\title{
Erratum to: Gender difference and effect of pharmacotherapy: findings from a smoking cessation service
}

\author{
N. J. Walker ${ }^{1 *}$, H. C. van Woerden ${ }^{2,3}$, V. Kiparoglou', Y. Yang ${ }^{4}$, H. Robinson ${ }^{5}$ and E. Croghan ${ }^{5}$
}

\section{Erratum}

Following publication of this article [1], it has come to our attention that a line has been omitted from the acknowledgements. Could you please add the line, "This research was supported by the NIHR Oxford Biomedical Research Centre".

\footnotetext{
Author details

'Oxford Biomedical Research Centre, Churchill Hospital, Oxford, England.

${ }^{2}$ Institute of Primary Care \& Public Health, Cardiff University, Cardiff, Wales. ${ }^{3}$ Centre for Health Sciences, University of the Highlands and Islands, Inverness, Scotland. ${ }^{4}$ Nuffield Department of Primary Care Health Science, University of Oxford, Oxford, England. ${ }^{5}$ Quit 51 Stop Smoking Service,

Burton-on-, Trent, England.
}

Received: 6 October 2016 Accepted: 29 March 2017

Published online: 04 April 2017

\section{Reference}

1. Walker, N., van Woerden, H., Kiparoglou, V., Yang, Y., Robinson, H. and Croghan, E. (2016). Gender difference and effect of pharmacotherapy: findings from a smoking cessation service. BMC Public Health.2016; 16:1038. doi: 10.1186/s12889-016-3672-y

\footnotetext{
*Correspondence: Neil.Walker3@ouh.nhs.uk

'Oxford Biomedical Research Centre, Churchill Hospital, Oxford, England
} 